\title{
Screen time: studying gene function in iPSCs
}

$\mathrm{i}^{3} \mathrm{~N}$ cells were
engineered to
express the
machinery
necessary
for CRISPR
interference
(CRISPRi)

Methods to reprogramme adult human somatic cells to produce induced pluripotent stem cells (iPSCs) and their differentiation into neurons have been optimized, presenting opportunities to investigate hard to access cell populations. Two new papers now combine iPSC technology with precision gene editing to better understand neuronal function and dysfunction.

CRISPR-Cas9 gene editing, in which the nuclease Cas9 is targeted by single-guide RNAs (sgRNAs) to a specific region of DNA, can be used to disrupt the function of individual genes and assess their biological roles. Gene editing can be applied in large-scale screens to investigate gene function. To date, these screens have usually been performed in cultured cancer or stem cell lines. However, many genes have multiple, cell type-specific roles, making it important to investigate their function in the cell type of interest.

To generate a neuronal genetic screening platform, Tian et al. used the ' $\mathrm{i}^{3} \mathrm{~N}$ ' iPSC cell line, which can be differentiated into a homogenous population of glutamatergic neurons. Here, the $i^{3} \mathrm{~N}$ cells were engineered to

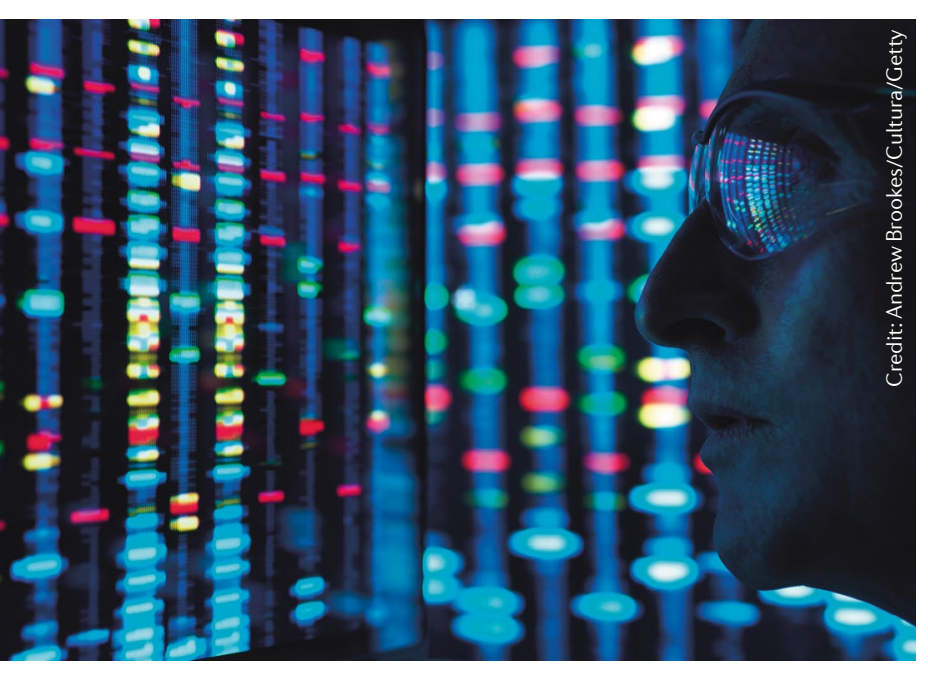

express the machinery necessary for CRISPR interference (CRISPRi), in which gene transcription is inhibited at target sites.

Tian et al. used the resulting 'CRISPRi-i ${ }^{3} \mathrm{~N}$ IPSCs' to perform a systematic screen for cell survival genes. They transduced the cells with a library of sgRNAs targeting 2,325 different genes. After differentiating the cells, they measured the numbers of neurons (or non-differentiated iPSCs) expressing each sgRNA to determine the effects of gene knockdown on survival. They identified a set of genes essential for the survival of both cell types, as well as many neuron-specific or iPSC-specific 'essential' survival genes.

Tian et al. extended their strategy in several ways to provide further insights. Using single-cell RNA sequencing, they revealed both gene-specific and cell type-specific effects of gene knockdown on the transcriptomes of the affected cells. They also developed tools that allowed the temporal regulation of CRISPRi-meditated gene knockdown and incorporated CRISPRi- $i^{3} \mathrm{~N}$ IPSCs into a high-content array screening platform to assess the effects of gene knockdown on cell morphology.

There is widespread hope that iPSC technology will facilitate the investigation of human nervous system diseases, particularly given its capacity to generate isogenic human neuronal cell lines carrying mutations in disease-related genes of interest. In their study, Kwart et al. used CRISPR-Cas9 gene editing to generate a panel of isogenic iPSC cell lines carrying mutations linked to familial forms of Alzheimer disease (fAD) to investigate their common phenotypic effects.

Many fAD cases are caused by mutations in genes involved in the metabolism of amyloid precursor protein (APP). Other studies have linked alterations in endocytic trafficking to the disease. Kwart et al. used gene editing to introduce seven different $\mathrm{fAD}$ mutations to human iPSCs, creating a public resource of isogenic mutant iPSC lines. Following their differentiation into cortical-like neurons, RNA sequencing was performed. This revealed a set of genes that were differentially expressed in all of the cell lines carrying mutations versus control lines, including a number of $\mathrm{AD}$-related and endocytosis-related genes. Furthermore, immunostaining revealed that an increase in endosome size was a common feature of the mutant neurons.

Dysregulated APP processing alters the production of its cleavage products, including $\beta \mathrm{C}$-terminal fragments ( $\beta$-CTFs). Kwart et al. showed that the accumulation of $\beta$-CTFs in fAD mutant neurons correlated with endosomal size, consistent with a previously suggested role for these fragments in endocytic dysfunction. Indeed, endosomal enlargement in mutant neurons was prevented by the inhibition of one of the key enzymes involved in APP processing ( $\beta$-secretase) and was promoted by the inhibition of the enzyme $\gamma$-secretase, which increases $\beta$-CTF levels.

These studies illustrate the power of two recently developed biological tools to dissect the mechanisms at work in neuronal health and disease.

Katherine Whalley

ORIGINAL ARTICLES Tian, R. et al. CRISPR interference-based platform for multimodal genetic screens in human iPSC-derived neurons. Neuron https://doi.org/10.1016/j.neuron. 2019.07.014 (2019) |Kwart, D. et al. A large panel of isogenic APP and PSEN1 mutant human iPSC neurons reveals shared endosomal abnormalities mediated by APP $\beta$-CTFs, not A $\beta$. Neuron https:// doi.org/10.1016/j.neuron.2019.07.010 (2019) 